\title{
Study of the Cathodic Protection by Printed Current System for the Buried Duct through the Finite Elements Technique
}

\author{
Fabiana O.R. Staroin, ${ }^{a}$ Anderson C. Mossi, ${ }^{\text {b }}$ Moisés M. Pariona a, \\ ${ }^{a}$ Pós-Graduação em Engenharia e Ciência de Materiais \\ ${ }^{b}$ Departamento de Matemática e Estatística, Universidade Estadual de Ponta Grossa-UEPG \\ Campus Uvaranas, Bloco CIPP, CEP 84030-900, Ponta Grossa-PR, Brasil
}

Received 19 April 2004; accepted 29 December 2004

\begin{abstract}
In the present study we have made the numeric simulation by finite elements of the cathodic protection by printed current system for the buried duct. The built model was based on the steel carbon type SAE-AISI 1015, and this steel is the constituent material of the duct. This work allowed analyzing the potential distribution in the proposed project and the parameters that more influence the variation of this potential. Those parameters were such as resistivity of the soil, distance between cathode and anode, position of the anode, potential and diameter of the cathode. As a result it was verified that the resistivity of the electrolyte and the distance cathode/anode influence significantly the distribution of the potential of the projected system of cathodic protection.
\end{abstract}

Keywords: printed current, cathodic protection, buried duct, finite elements.

\section{Introdução}

Um grande investimento é realizado em estruturas enterradas, tais como tubulações de condução de água, combustíveis líquidos e gasosos, tanques de armazenagem etc., sendo este um problema de preocupação mundial. Quando estes elementos metálicos estão em contato direto com o solo e sem proteção, eles são sujeitos à corrosão. A corrosão é um dos problemas que atualmente

\footnotetext{
* Corresponding author. E-mail address: mmpariona@uepg.br
} 
exige muita cautela. Por exemplo, há uma grande preocupação com a preservação do meio ambiente, pois muitos acidentes ambientais ocorrem devido ao vazamento de óleo, gás, etc. [1-4].

A proteção catódica por corrente impressa é uma técnica utilizada para evitar este tipo de problema em estruturas metálicas e teve sua primeira aplicação aproximadamente em 1910-1912 [1]. Hoje, é aplicada em muitas estruturas metálicas como navios, tubulações, e outras. Para uma proteção catódica bem projetada, faz-se necessário aplicar adequadamente métodos experimentais de controlo, os quais podem ser auxiliados pela modelagem matemática do sistema com simulação do fenômeno corrosivo.

Através da modelagem matemática pode-se prever a influência de certos parâmetros referentes ao eletrólito e material a ser protegido, no que diz respeito ao processo corrosivo, assim como gerar economia na tomada de decisões $[5,6$, 7]. A simulação numérica por elementos finitos pode prever e controlar os fenômenos, podendo-se comparar com resultados experimentais para sua validade. A economia de tempo e material é uma das grandes vantagens que oferece a simulação numérica.

A aplicação da simulação numérica no sistema de proteção catódica é um assunto de grande interesse na pesquisa científica $[8,9]$, baseado numa análise precisa do comportamento da corrosão onde a preocupação está em analisar o potencial e a densidade de corrente na superfície do catodo. Segundo este autor o método de elementos finitos pode ser também utilizado para o estudo de fluxo de fluidos, transferência de calor, mecânica de deformação, etc.

$\mathrm{Na}$ maioria da literatura revisada considera-se o modelo matemático da equação de Laplace, sendo este modelo o que descreve melhor o problema de proteção catódica quando não existe densidade volumétrica de carga ou fontes de corrente, além do modelo utilizado, pois depende das condições de contorno consideradas [10-14].

Neste trabalho, a simulação numérica da proteção catódica por corrente impressa do gasoduto foi realizada utilizando o método de elementos finitos através do 
software Ansys ${ }^{\mathrm{MR}}$, que é um programa de elementos finitos, e um computador Pentium III $1 \mathrm{GHz}$.

O objetivo deste trabalho foi analisar e identificar a influência das variáveis, tais como, resistividade do solo, diâmetro do tubo (catodo), posição dos anodos, potencial do catodo e distância anodo/cátodo, na distribuição de potencial em 3D, sendo que a magnitude desta distribuição é importante no controle da proteção catódica. Para esta finalidade, primeiro foi realizado o projeto de proteção catódica baseado na montagem do planejamento fatorial das variáveis envolvidas e logo foram calculadas todas as grandezas elétricas envolvidas no sistema. Finalmente, a análise dos resultados apresentados foi realizada em forma qualitativa e quantitativa da simulação numérica.

\section{Material e método}

Antes da execução da simulação foi projetada a proteção catódica através da técnica de corrente impressa, com o objetivo de ter um resultado mais representativo e realístico. O tubo de aço a ser protegido corresponde ao aço AISI 1015 (aço carbono de baixa liga). O primeiro passo foi determinar a dimensão geométrica do tubo para a simulação. Preestabeleceu-se uma tubulação de aço de 12,5 m considerando a distância máxima e mínima entre cátodo e anodo de $25 \mathrm{~m}$ e $5 \mathrm{~m}$, respectivamente, e considerou-se o sistema catodo/anodo numa profundidade de $1,5 \mathrm{~m}$ da superfície do solo. Para as características dimensionais citadas anteriormente foi projetado o sistema de proteção catódica por corrente impressa, sendo os cálculos da intensidade de corrente necessária para a proteção abordados a seguir.

\section{Variáveis físicas e geométricas do solo e do tubo (apresentadas na Tabela 1)}

Para o cálculo da corrente de proteção considerou-se a equação (1) [1]; para isto, considerou-se também um fator de revestimento de $\mathrm{Rev}=0.2$ (tubo praticamente nu) e fator de velocidade ( $\left.f^{*}\right)$ igual a uma unidade, devido ao fato de não haver movimento do eletrólito em torno da tubulação: 


$$
\mathrm{I}=\text { S.d.f*. }(1-\operatorname{Re} v)
$$

onde $d=73.73-13.35 \log \lambda$, sendo $\lambda$ a resistividade do solo. Logo, obteve-se os valores da corrente descritos na penúltima coluna da Tabela 2 para todas as combinações possíveis da Tabela 1 .

Tabela 1. Variáveis físicas e geométricas do solo e do tubo utilizados na simulação.

\begin{tabular}{lcc}
\hline Variáveis & $\begin{array}{c}\text { Valores mínimos } \\
\text { considerados }\end{array}$ & $\begin{array}{c}\text { Valores máximos } \\
\text { considerados }\end{array}$ \\
\hline Resistividade do solo $(\Omega . c m)$ & 5000 & 50000 \\
Diâmetro externo do tubo $(\mathrm{m})$ & 0.111 & 0.275 \\
\hline
\end{tabular}

Tabela 2. Cálculo da corrente de proteção e massa do ânodo.

\begin{tabular}{|c|c|c|c|c|}
\hline $\begin{array}{c}\lambda \\
\text { (resistividade) } \\
(\Omega . \mathrm{cm})\end{array}$ & $\begin{array}{c}d=(73.73-13.35 \log \lambda) \times 10^{-3} \\
\left(\mathrm{~A} / \mathrm{m}^{2}\right)\end{array}$ & $\begin{array}{c}\mathrm{S} \text { (superfície a } \\
\text { proteger) } \\
\left(\mathrm{m}^{2}\right)\end{array}$ & $\begin{array}{c}\text { corrente de } \\
\text { proteção } \\
\mathrm{I}=\mathrm{S} \times \mathrm{d} \times(1-0,2)\end{array}$ & $\begin{array}{c}\text { massa do } \\
\text { anodo } \\
(\mathrm{kg})\end{array}$ \\
\hline 5000 & $24.34 \times 10^{-3}$ & 4.3589 & 0.08487 & 0.6601 \\
\hline 5000 & $24.34 \times 10^{-3}$ & 4.3589 & 0.03835 & 0.2982 \\
\hline 50000 & $10.998 \times 10^{-3}$ & 10.7992 & 0.21028 & 1.6355 \\
\hline 50000 & $-10.998 \times 10^{-3}$ & 10.7992 & 0.09501 & 0.7389 \\
\hline
\end{tabular}

\section{Cálculo do número e dimensão de ânodos}

Foi realizado através da equação (2) [1]

$$
M=\frac{\text { dg.t.I }}{f}
$$

onde: $\mathrm{M}$ - massa necessária do anodo $(\mathrm{kg}), \mathrm{dg}$ - desgaste do anodo $\mathrm{kg} /($ A.ano $)$, t - tempo (ano), I - corrente de proteção (A), f - fator de utilização. 
Determinou-se a massa necessária do anodo para cada intensidade de corrente calculada anteriormente. Para proteger $12.5 \mathrm{~m}$ de tubulação, utilizou-se um fator $f=0.9$, ideal para materiais com uma forma geométrica tubular linear. A liga FeSi foi considerada como anodo com o desgaste de $0.35 \mathrm{~kg} /($ A.ano $)$ e o tempo de vida útil desejado da tubulação foi de 20 anos. Portanto de acordo com os dados da Tabela 2 e da equação (2), calculou-se a massa necessária, a qual, encontra-se descrita na última coluna da Tabela 2.

Através da última coluna da Tabela 2, observa-se que um anodo de aproximadamente $1.7 \mathrm{~kg}$, seria suficiente para proteger $12.5 \mathrm{~m}$ de cada uma das tubulações consideradas. Para o ânodo de ferro-silício (FeSi), foi considerada sua massa de $1.7 \mathrm{~kg}$ e sua densidade de massa de $7 \mathrm{~g} / \mathrm{cm}^{3}$. Baseado nestes dados foi determinada a dimensão geométrica da barra do ânodo, sendo o volume do anodo calculado através de:

$$
\text { volume }=\frac{1700 \mathrm{~g}}{7 \mathrm{~g} / \mathrm{cm}^{3}}=242.85 \mathrm{~cm}^{3}
$$

Portanto, considerando o anodo com $5 \mathrm{~cm}(0.05 \mathrm{~m})$ de diâmetro, obteve-se o comprimento do anodo utilizando a equação seguinte, ou seja, o volume de um cilindro:

$$
\text { Comprimento }=\frac{242.85}{\pi \times 2.5^{2}}=12.36 \mathrm{~cm}=0.1236 \mathrm{~m}
$$

Obteve-se então um anodo cilíndrico de $0.05 \mathrm{~m}$ de diâmetro e $0.1236 \mathrm{~m}$ de comprimento. A geometria considerada para a simulação está representada na Fig. 1.

Cálculo da resistência do circuito do sistema considerando $1 / 4$ de simetria do modelo descrito anteriormente

A) As resistências para os dois tipos de catodos com diâmetro menor e maior foram calculadas através da eq. [1] (3): 


$$
R c^{\prime}=\frac{78.49 \cdot \lambda}{P}
$$

onde $\mathrm{P}$ é a massa do tubo (catodo) em $\mathrm{kg} / \mathrm{m}$ linear, $\lambda$ é a resistividade do catodo em $\mu \Omega . c m$ e $\mathrm{Rc}^{\prime}$ é a resistência do catodo.
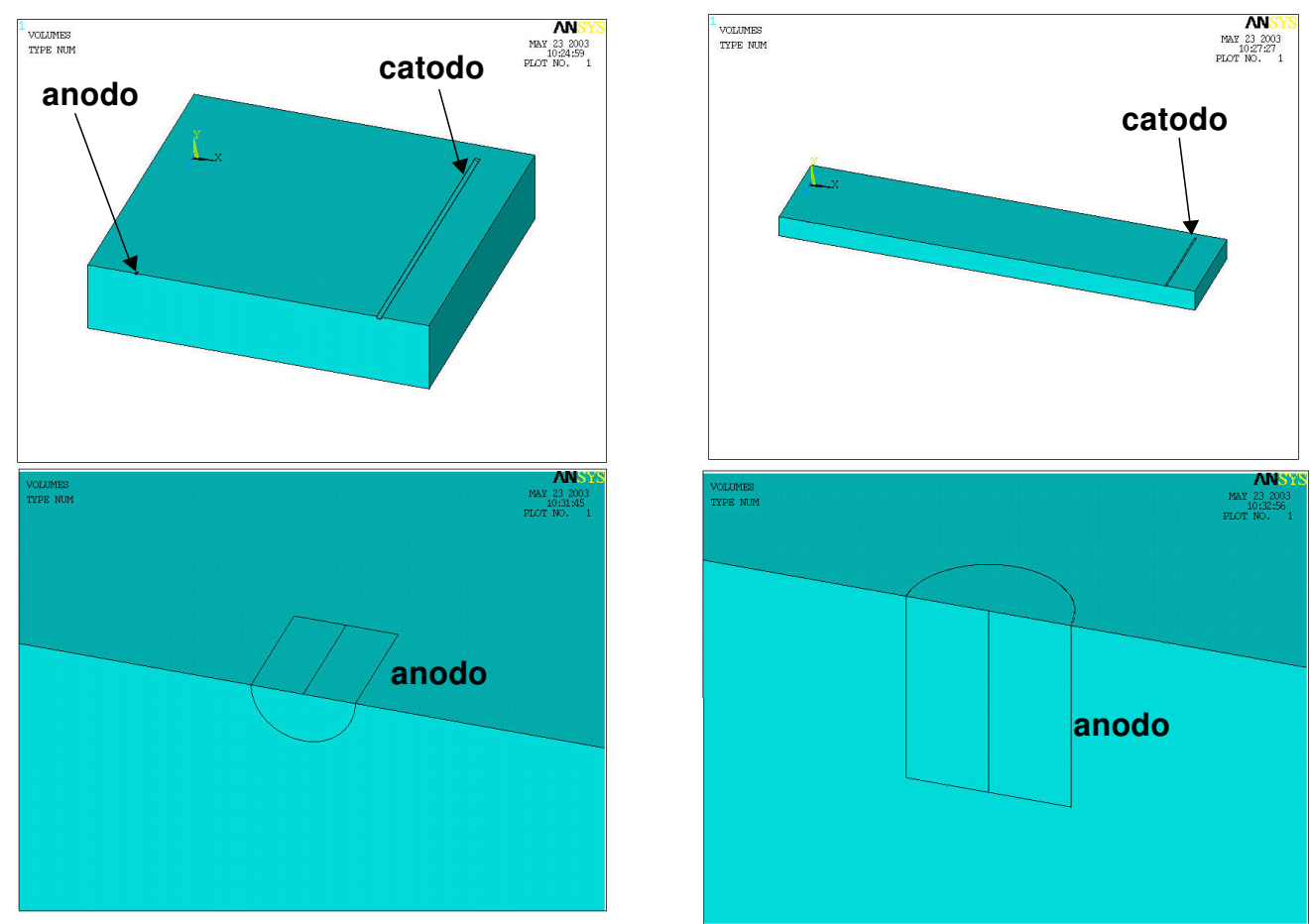

Figura 1. Representação geométrica dos modelos usados para a simulação com anodo na posição horizontal.

Tabela 3. Propriedades da tubulação (catodo).

\begin{tabular}{lcc}
\hline Propriedades & $\begin{array}{c}\text { Tubulação de diâmetro } \\
\text { menor }(0.111 \mathrm{~m})\end{array}$ & $\begin{array}{c}\text { Tubulação de diâmetro maior } \\
(0.275 \mathrm{~m})\end{array}$ \\
\hline Comprimento $(\mathrm{m})$ & 6.25 & 6.25 \\
Densidade de massa $\left(\mathrm{g} / \mathrm{cm}^{3}\right)$ & 7.87 & 7.87 \\
Massa $(\mathrm{kg})$ & 38.58 & 154.99 \\
Volume $\left(\mathrm{cm}^{3}\right)$ & 4902.5 & 19695 \\
Resistividade $(\Omega . \mathrm{m})$ & $1.59 \times 10^{-7}$ & $1.59 \times 10^{-7}$ \\
P $(\mathrm{kg} / \mathrm{m})$ & 6.1728 & 24.7999 \\
Resistência $(\Omega)$ & $1263.59 \times 10^{-6}$ & $314.5 \times 10^{-6}$ \\
\hline
\end{tabular}

Para uma melhor compreensão dos parâmetros utilizados e calculados, descrevese na Tabela 3 as propriedades da tubulação (catodo), onde na última linha 
encontram-se as resistências calculadas pela eq. (3) para os dois tamanhos de catodo.

B) Resistência da interface anodo/eletrólito.

Para anodos na posição vertical, com diâmetro de $5 \mathrm{~cm}$ e comprimento de 6.18 $\mathrm{cm}$, e ainda, considerando a resistividade mínima e máxima foram feitas os respectivos cálculos através da eq. [1] (4).

$$
R_{v}=\frac{\lambda}{2 \pi c} \cdot\left(\ln \frac{8 c}{\Pi}-1\right)
$$

onde: $R_{v}=$ resistência de contato de um anodo vertical, em ohm, $\lambda=$ resistividade do eletrólito, em ohm.cm, $\mathrm{c}=$ comprimento do anodo, em $\mathrm{m}, \Pi=$ dobro da profundidade em que a tubulação está enterrada, em cm.

Para anodos na posição horizontal com diâmetro e comprimento de $5 \mathrm{~cm}$ e 6.18 cm respectivamente, considerando a resistividade mínima e máxima realizaramse cálculos através da eq. (5).

$$
\mathrm{R}_{\mathrm{h}}=\frac{\lambda}{2 \pi \mathrm{c}}\left(\ln \frac{4 \mathrm{c}^{2}+4 \mathrm{c} \sqrt{\Pi^{2}+\mathrm{c}^{2}}}{\theta \Pi}+\frac{\Pi}{\mathrm{c}}-\frac{\sqrt{\Pi^{2}+\mathrm{c}^{2}}}{\mathrm{c}}-1\right)
$$

onde: $\mathrm{R}_{\mathrm{h}}=$ resistência de contato de um anodo horizontal, em ohm, $\lambda=$ resistividade do eletrólito, em ohm.cm, $\mathrm{c}=$ comprimento do anodo, em $\mathrm{cm}, \Pi=$ dobro da profundidade em que o anodo está enterrado, em $\mathrm{cm}, \theta=$ diâmetro do anodo, em $\mathrm{cm}$.

Para uma melhor compreensão dos parâmetros utilizados e calculados, descrevese na Tabela. 4 as características do anodo e nas duas últimas linhas encontramse as resistências respectivas calculadas pelas eqs. (4) e (5). 
Tabela 4. Propriedades da liga FeSi (anodo).

\begin{tabular}{|c|c|c|}
\hline Propriedades & Anodo vertical & Anodo horizontal \\
\hline Diâmetro $(\mathrm{m})$ & 0.05 & 0.05 \\
\hline Comprimento $(\mathrm{m})$ & 0.1236 & 0.1236 \\
\hline Densidade de massa $\left(\mathrm{g} / \mathrm{cm}^{3}\right)$ & 7 & 7 \\
\hline Massa $(\mathrm{kg})$ & 1.7 & 1.7 \\
\hline Volume $\left(\mathrm{cm}^{3}\right)$ & 242.85 & 242.85 \\
\hline Resistividade & $5 \times 10^{-7}$ & $5 \times 10^{-7}$ \\
\hline $\begin{array}{c}\text { Resistência (interface anodo/eletrólito) } \\
\lambda=5000 \Omega . \mathrm{cm} \Pi=300 \mathrm{~cm}\end{array}$ & 127.83 & 84.50 \\
\hline
\end{tabular}

C) Resistência da interface catodo/eletrólito.

As resistências da interface catodo/eletrólito para os dois catodos, com diâmetros menor e maior, foram calculadas através da eq. (6):

$$
R c=\frac{\lambda}{2 \pi c(1-0.2)}\left(\ln \frac{4 c^{2}+4 c \sqrt{\Pi^{2}+c^{2}}}{\theta \Pi}+\frac{\Pi}{c}-\frac{\sqrt{\Pi^{2}+c^{2}}}{c}-1\right)
$$

onde: $\mathrm{R}_{\mathrm{c}}=$ resistência de contato de um catodo, em ohm, $\lambda=$ resistividade do eletrólito, em ohm.cm, $\mathrm{c}=$ comprimento do catodo, em $\mathrm{cm}, \Pi=$ dobro da profundidade em que o catodo está enterrado, em cm, $\theta=$ diâmetro do anodo, em $\mathrm{cm}$.

Para uma melhor compreensão dos cálculos obtidos, construiu-se a Tabela 5, onde se descrevem os parâmetros da equação (6) e seus respectivos cálculos, considerando $\Pi=300 \mathrm{~cm}$.

Tabela 5. Cálculo da resistência catodo/eletrólito.

\begin{tabular}{|c|c|c|}
\hline $\begin{array}{c}\text { Resistividade do } \\
\text { eletrólito }\end{array}$ & $\begin{array}{c}\text { Tubulação } \\
\text { diâmetro: } 11.10 \mathrm{~cm} \\
\text { comprimento: } 625 \mathrm{~cm}\end{array}$ & $\begin{array}{c}\text { Tubulação } \\
\text { diâmetro: } 27.5 \mathrm{~cm} \\
\text { comprimento: } 625 \mathrm{~cm}\end{array}$ \\
\hline $5000 \Omega . c m$ & $5.1376 \Omega$ & $4.4157 \Omega$ \\
\hline $50000 \Omega . c m$ & $51.3769 \Omega$ & $44.1573 \Omega$ \\
\hline
\end{tabular}


D) Para o cálculo da resistência total utilizou-se a eq. (7):

$$
\mathrm{R}_{\mathrm{t}}=\mathrm{R}_{\mathrm{c}}^{\prime}+\mathrm{R}_{\mathrm{c}}+\mathrm{R}_{\mathrm{n}}
$$

A Tabela 6 apresenta a resistência total calculada através dos valores calculados nas Tabelas 3, 4 e 5 .

Tabela 6. Cálculo da resistência total do sistema.

\begin{tabular}{lccc}
\hline Posição do anodo & Diâmetro da tubulação & Resistividade do solo & $\begin{array}{c}\text { Resistência total } \\
\mathrm{R}_{\mathrm{c}}^{\prime}+\mathrm{R}_{\mathrm{c}}+\mathrm{R}_{\mathrm{n}}\end{array}$ \\
\hline Vertical & $0.111 \mathrm{~m}$ & $\lambda=5000 \Omega . \mathrm{cm}$ & $132.9688 \Omega$ \\
Vertical & $0.111 \mathrm{~m}$ & $\lambda=5000 \Omega . \mathrm{cm}$ & $132.2460 \Omega$ \\
Vertical & $0.275 \mathrm{~m}$ & $\lambda=50000 \Omega . \mathrm{cm}$ & $1329.6881 \Omega$ \\
Vertical & $0.275 \mathrm{~m}$ & $\lambda=5000 \Omega \cdot \mathrm{cm}$ & $1322.4676 \Omega$ \\
Horizontal & $0.111 \mathrm{~m}$ & $\lambda=5000 \Omega . \mathrm{cm}$ & $89.6388 \Omega$ \\
Horizontal & $0.111 \mathrm{~m}$ & $\lambda=5000 \Omega . \mathrm{cm}$ & $88.9160 \Omega$ \\
Horizontal & $0.275 \mathrm{~m}$ & $\lambda=50000 \Omega \cdot \mathrm{cm}$ & $896.4581 \Omega$ \\
Horizontal & $0.275 \mathrm{~m}$ & $\lambda=50000 \Omega . \mathrm{cm}$ & $889.2376 \Omega$ \\
\hline
\end{tabular}

Otimização do sistema de proteção catódica por corrente impressa

No próximo passo tomado, foram definidas previamente as propriedades físicas e geométricas que intervem no sistema de proteção catódica. Estas propriedades são apresentadas na Tabela 7, que descreve seus respectivos valores máximos e mínimos.

Na Tabela 8, representa-se os parâmetros dependentes que envolvem o projeto do sistema de proteção catódica por corrente impressa.

Tabela 7. Planejamento dos parâmetros independentes e seus respectivos valores considerados.

\begin{tabular}{cccc}
\hline Simbologia & Parâmetros independentes & Nível inferior $(-1)$ & Nível superior $(+1)$ \\
\hline 1 & Resistividade do solo & $5000 \mathrm{ohm} . \mathrm{cm}$ & $50000 \mathrm{ohm} . \mathrm{cm}$ \\
2 & Diâmetro do tubo (catodo) & $0.111 \mathrm{~m}$ & $0.275 \mathrm{~m}$ \\
3 & Posição dos anodos & Horizontal & Vertical \\
4 & Potencial do catodo & $-0.85 \mathrm{~V}$ & $-1.2 \mathrm{~V}$ \\
5 & Distância anodo/catodo & $5 \mathrm{~m}$ & $25 \mathrm{~m}$ \\
\hline
\end{tabular}


Tabela 8. Parâmetros dependentes para o projeto de proteção catódica.

\begin{tabular}{cl}
\hline Simbologia & \multicolumn{1}{c}{ Parâmetros dependentes } \\
\hline $\mathrm{A}$ & Densidade de corrente de proteção $\left(\mathrm{em} \mathrm{A} / \mathrm{cm}^{2}\right)$ \\
$\mathrm{B}$ & Resistência do sistema $\left(\mathrm{R}_{\mathrm{t}}\right)(\mathrm{em} \Omega)$ \\
$\mathrm{C}$ & Diferença de potencial $\left(\Delta \mathrm{V}=1.2 \times R_{t} \times \mathrm{I}=1.2 \times A \times B\right)(\mathrm{em}$ volts $)$ \\
$\mathrm{D}$ & Potencial do anodo $(\mathrm{Va}=\mathrm{Vc}+\Delta \mathrm{V})(\mathrm{em}$ volts $)$ \\
\hline
\end{tabular}

A seguir, foi realizado um planejamento fatorial em dois níveis dos cinco parâmetros independentes citados que envolvem o projeto de proteção catódica, conseqüentemente foram geradas 32 linhas através deste planejamento de que foram mostrados os cálculos dos parâmetros dependentes [15]. Com a finalidade de reduzir o trabalho de simulação, foi realizado um planejamento fatorial fracionário de resolução $\mathrm{V}$, ou seja, $2_{\mathrm{V}}^{5}{ }^{1}=2^{4}=16$. Para gerar este planejamento fracionário foi considerado que a variável 5 é equivalente a $(1 \times 2 \times 3 \times 4)$, ou seja, cujo resultado é apresentado na Tabela 9. Portanto, através deste último planejamento realizou-se a simulação para cada linha desta tabela. Como resultado desta simulação foi mostrada a distribuição de potencial através do software ANSYS baseado em elementos finitos. Para isto, foram aplicadas as condições de contorno ao sistema, tais como, potencial do catodo e anodo, corrente de proteção, potencial no contorno do eletrólito nulo, tendo-se nesta circunstância considerado o eletrólito homogêneo [15].

\section{Resultado e discussão}

De acordo com cada linha da Tabela 9, realizou-se a simulação numérica através do software Ansys baseado em elementos finitos. Para a realização da simulação, primeiramente atribuiu-se aos materiais considerados suas propriedades, ou seja, a resistividade elétrica do eletrólito, catodo e anodo. Como condição de contorno, foram impostos ao sistema os seguintes parâmetros: densidade de corrente de proteção aplicada no anodo; potencial no catodo, no anodo e entorno do eletrólito [15]. 
Tabela 9. Planejamento fatorial fracionário $2{ }_{\mathrm{v}}{ }^{5-1}$ baseado nas Tabelas 7 e 8 .

\begin{tabular}{|c|c|c|c|c|c|c|c|c|c|}
\hline & \multicolumn{5}{|c|}{ Parâmetros independentes } & \multicolumn{4}{|c|}{ Parâmetros dependentes } \\
\hline & 1 & 2 & 3 & 4 & 5 & $\overline{\mathrm{A}}$ & $\bar{B}$ & $\mathrm{C}$ & $\bar{D}$ \\
\hline & (ohm.m) & $(\mathrm{m})$ & $\mathrm{H} / \mathrm{V}$ & (Volts) & $(\mathrm{m})$ & $\mathrm{A} / \mathrm{m}^{2}$ & (ohm) & (Volts) & (Volts) \\
\hline $1^{a}$ & -1 & -1 & -1 & -1 & -1 & 1.984981 & 89.6388 & 9.129174 & 8.279174 \\
\hline $2^{a}$ & +1 & +1 & -1 & -1 & -1 & 2,222135 & 889,2376 & 101,3838 & 100,5338 \\
\hline $3^{a}$ & +1 & -1 & +1 & -1 & -1 & 0,896974 & 1329,6881 & 61,19225 & 60,34225 \\
\hline $4^{a}$ & -1 & +1 & +1 & -1 & -1 & 4,918016 & 132,2460 & 33,37043 & 32,52043 \\
\hline $5^{a}$ & +1 & -1 & -1 & +1 & -1 & 0,896974 & 896,4581 & 41,255 & 40,055 \\
\hline $6^{a}$ & -1 & +1 & -1 & +1 & -1 & 4,918016 & 88,9160 & 22,43671 & 21,23671 \\
\hline $7^{a}$ & -1 & -1 & +1 & +1 & -1 & 1,984981 & 132,9688 & 13,54207 & 12,34207 \\
\hline $8^{a}$ & +1 & +1 & +1 & +1 & -1 & 2,222135 & 1322,4676 & 150,7772 & 149,5772 \\
\hline $9^{a}$ & +1 & -1 & -1 & -1 & +1 & 0,896974 & 896,4581 & 41,255 & 40,405 \\
\hline $10^{a}$ & -1 & +1 & -1 & -1 & +1 & 4,918016 & 88,9160 & 22,43671 & 21,58671 \\
\hline $11^{a}$ & -1 & -1 & +1 & -1 & +1 & 1,984981 & 132,9688 & 13,54207 & 12,69207 \\
\hline $12^{a}$ & +1 & +1 & +1 & -1 & +1 & 2,222135 & 1322,4676 & 150,7772 & 149,9272 \\
\hline $13^{a}$ & -1 & -1 & -1 & +1 & +1 & 1,984981 & 89,6388 & 9,129174 & 7,929174 \\
\hline $14^{a}$ & +1 & +1 & -1 & +1 & +1 & 2,222135 & 889,2376 & 101,3838 & 100,1838 \\
\hline $15^{a}$ & +1 & -1 & +1 & +1 & +1 & 0,896974 & 1329,6881 & 61,19225 & 59,99225 \\
\hline $16^{a}$ & -1 & +1 & +1 & +1 & +1 & 4,918016 & 132.2460 & 33,37043 & 32,17043 \\
\hline
\end{tabular}

De acordo com as simulações de cada linha da Tabela 9, observou-se variação significativa nos resultados que correspondem às linhas 1, 7 e 13, que apresentaram uma maior variação na distribuição de potencial do sistema. Por outro lado, no resultado da simulação que corresponde à linha 8, ocorreu uma menor distribuição de potencial. Portanto, com o objetivo de discutir os resultados das simulações que correspondem às linhas 1, 7, 8, 11 e 13, seus parâmetros independentes e dependentes estão descritos com maiores detalhes na Tabela 10.

Primeiramente, analisou-se o resultado da simulação que corresponde à $1^{\text {a }}$ linha, cuja distribuição de potencial encontra-se ilustrada na Fig. 2. Ao analisar o diagrama de potenciais nesta figura, identificam-se os valores de potenciais por meio de escala de cores.

Como também, o resultado é mostrado quantitativamente, onde o potencial é apresentado em vários pontos do sistema. Pode-se verificar na Fig. 2 (a), que o valor do potencial no anodo e catodo são respectivamente 8,279 e $-0,85$ volts, que concorda justamente com a Tabela 9. Observa-se, com mais detalhes no 
diagrama da Fig. 2 (b), uma queda de potencial em torno do anodo, sendo esta queda mais lenta no sentido do catodo.

Tabela 10. Parâmetros que envolvem as linhas 1, 7, 8, 11 e 13 da Tabela 9.

\begin{tabular}{l|c|c|c|c|c}
\hline \multicolumn{1}{c|}{ Características } & $1^{\mathrm{a}}$ linha & $7^{\star}$ linha & $8^{\star}$ linha & $11^{\star}$ linha & $13^{\star}$ linha \\
\hline Resistividade (ohm.m) & 50 & 50 & 500 & 50 & 50 \\
Diâmetro (duto) (m) & 0.111 & 0.111 & 0.275 & 0.111 & 0.111 \\
Posição (anodo) & Horizontal & Vertical & Vertical & Vertical & Horizontal \\
Potencial (duto) (volts) & -0.85 & -1.20 & -1.20 & -0.85 & -1.20 \\
Distância (anodo/duto) & 5 & 5 & 5 & 25 & 25 \\
Densidade de corrente & 1.98498 & 1.98498 & 2.22219 & 1.98498 & 1.98498 \\
Potencial (anodo) (volts) & 8.279 & 12.342 & 149.577 & 12.6920 & 7.929 \\
\hline
\end{tabular}

Já na Fig. 2 (c), verifica-se o potencial no próprio catodo e nas proximidades do cátodo; porém, mostra pouca variação significativa. Este fato ocorreu em todas as simulações, com exceção da simulação da linha 13, cuja escala de cores da distribuição de potencial nas proximidades do catodo foi diferente; porém, esta magnitude foi muita pequena e será discutida depois.

Por intermédio dos cálculos e das simulações realizadas, observou-se uma grande variação da distribuição de potencial que corresponde à $1^{\text {a }}$ linha. Em princípio, poder-se-ia atribuir este fato principalmente ao fator resistividade do eletrólito, porém este procedimento não seria o correto, pois existem outros parâmetros envolvidos no sistema.

Portanto, para realizar uma análise mais minuciosa de outros parâmetros, discutese a seguir uma comparação dos resultados das simulações que correspondem às linhas 1 e 8 , cujas características físicas e geométricas encontram-se descritas na Tabela 10.

A Fig. 3 representa a distribuição de potencial que corresponde à linha 8. Nota-se que a diferença do potencial entre catodo e anodo na Fig. 3 (a) é maior, comparada com a simulação ilustrada na Fig. 2 (a); isto pode ser verificado para a linha 8 , onde o anodo e catodo encontram-se com potencial de 149,58 e -1,2 volts, respectivamente. 

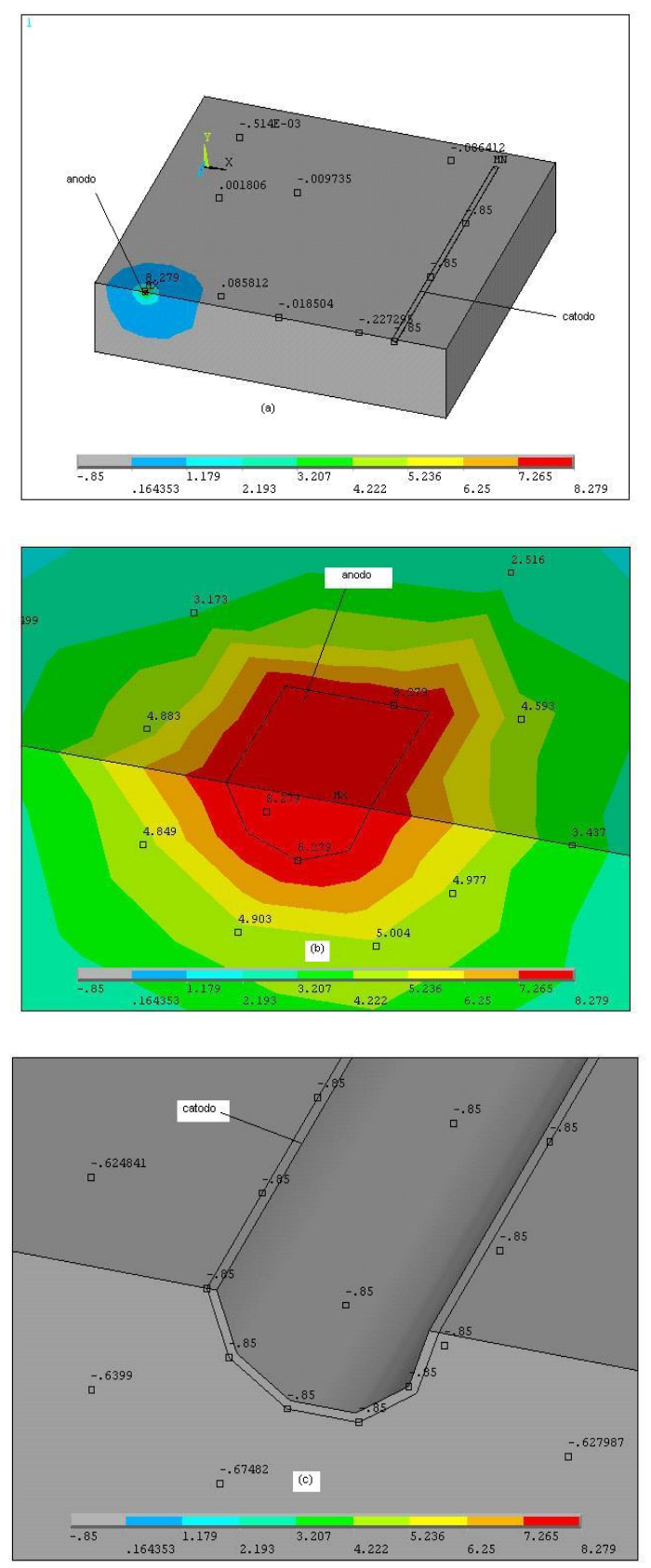

Figura 2. Diagrama da distribuição de potencial entre catodo e ânodo: (a) para a combinação dos parâmetros $(-1,-1,-1,-1,-1)$ da $1^{\text {a. }}$ linha, (b) distribuição de potencial com destaque próximo ao anodo, (c) distribuição de potencial com destaque no catodo.

Porém, a distribuição do potencial se estende mais no sistema que corresponde à linha 1 em comparação com a linha 8. Também é de notar que apenas o parâmetro distância anodo/catodo para ambas as linhas é comum. Portanto, podese dizer que esta característica pode ocorrer, devido ao efeito de qualquer um dos 
quatro parâmetros principais (resistividade do eletrólito, diâmetro do duto, posição do anodo e potencial do catodo) ou devido à interação conjunta deles.
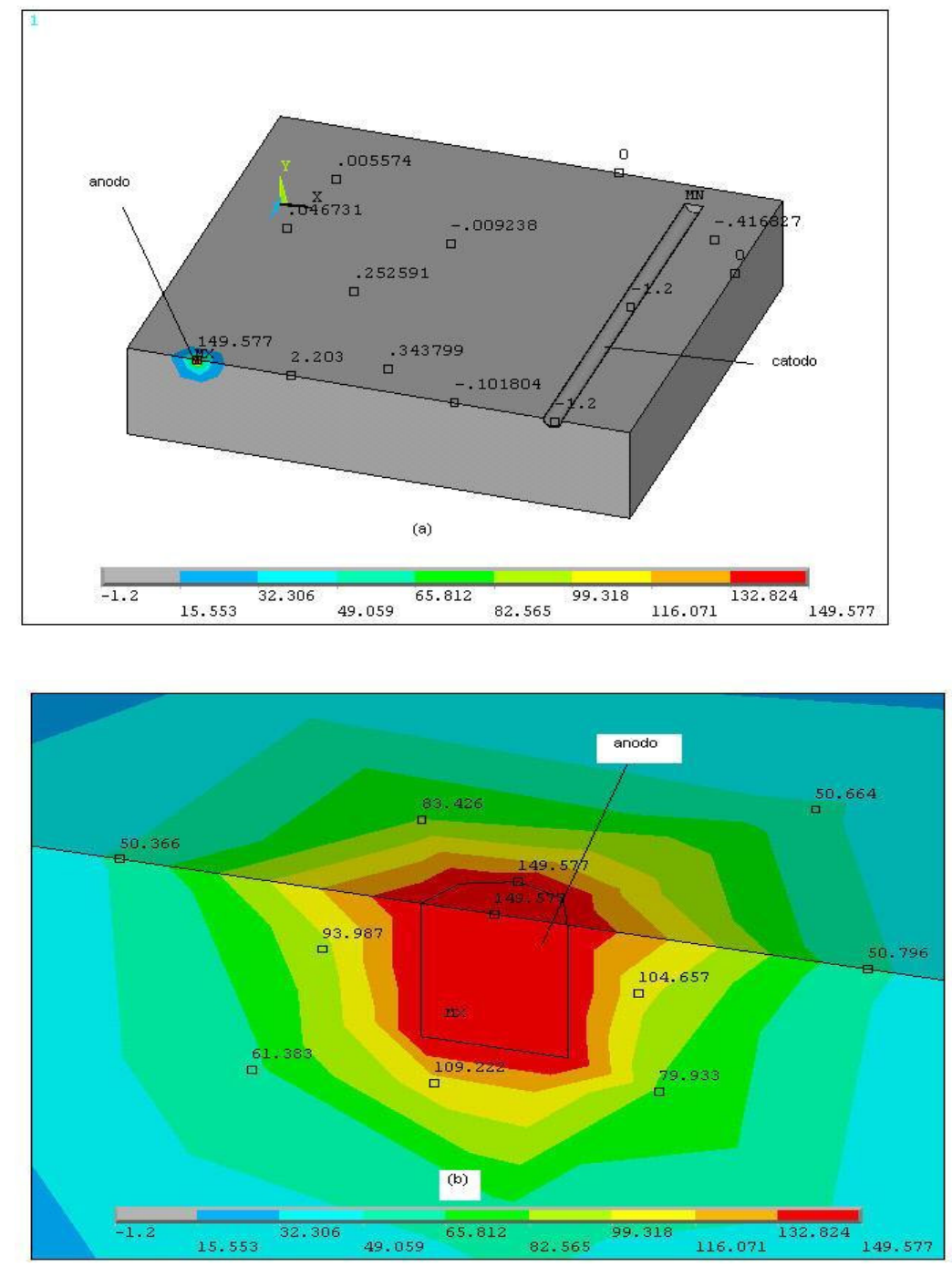

Figura 3. Diagrama da distribuição de potencial entre catodo e ânodo: (a) para combinação dos parâmetros $(+1,+1,+1,+1,-1)$ da $8^{\text {a. }}$ linha; (b) distribuição de potencial com destaque próximo ao anodo para a mesma linha.

No resultado da simulação que corresponde às linhas 1 e 8 , a posição do anodo é um dos parâmetros diferentes, sendo que o anodo da Fig. 3 (b), que corresponde à linha 8, encontra-se na posição vertical; possivelmente este parâmetro pode provocar uma maior variação da distribuição de potencial. 
Portanto, para esclarecer o efeito dos parâmetros sobre a distribuição de potencial, utilizar-se-ão ainda os resultados da simulação que corresponde às linhas 7 e 13, que também apresentaram uma distribuição de potencial significativa.

As Fig. 4 (a) e 4 (b) descrevem as distribuições de potenciais das linhas 7 e 13, respectivamente, e pode-se observar o seguinte:

- a distribuição de potencial que corresponde à linha 7, apresentada na Fig. 4 (a), teve um comportamento semelhante com a distribuição de potencial que corresponde à linha 1, ilustrada na Fig. 2 (a). Além disso, observando-se a Tabela 10, verifica-se nas linhas 1 e 7, que a resistividade do eletrólito, o diâmetro do duto e a distância entre catodo e anodo são os parâmetros comuns a estas linhas. Portanto, poder-se-ia dizer que a interação destes parâmetros influencia significativamente a distribuição de potencial do sistema nos resultados das simulações das linhas 1 e 7. Porém, os parâmetros posição do anodo e potencial do catodo são diferentes para estas linhas.

- a distribuição de potencial na simulação da linha 13, Fig. 4 (b), apresentou uma distribuição de potencial significativa no eletrólito. Porém, a magnitude desta distribuição de potencial varia na faixa de -0.18 a 0.83 V no eletrólito. Sendo esta magnitude muito pequena, conseqüentemente, teria um efeito desprezível na polarização do catodo, além disso, a distância catodo/anodo é grande.

- Observando nas linhas 1 e 13 da Tabela 10, verifica-se que a resistividade do eletrólito, o diâmetro do duto e a posição do anodo são parâmetros em comum a estas linhas; portanto também poderíamos dizer que são estes parâmetros que influenciam na maior distribuição de potencial do sistema nos resultados que correspondem às linhas 1 e 13. Também se observa, no resultado da simulação da linha 13, que a distribuição de potencial nas proximidades do anodo aparenta ser menor quando a distância entre anodo e catodo for maior.

Porém, ao visualizar com mais detalhe o anodo apresentado na Fig. 4 (d), observa-se que a distribuição de potencial neste caso é semelhante ao resultado da simulação do anodo da linha 1, Fig. 2 (b), ou seja, a magnitude do potencial 
nas proximidades do anodo para a simulação das linhas 13 e 1, variou respectivamente na faixa de $0.82 \mathrm{~V}$ a $7.279 \mathrm{~V}$ e $0.16 \mathrm{~V}$ a $8.29 \mathrm{~V}$.
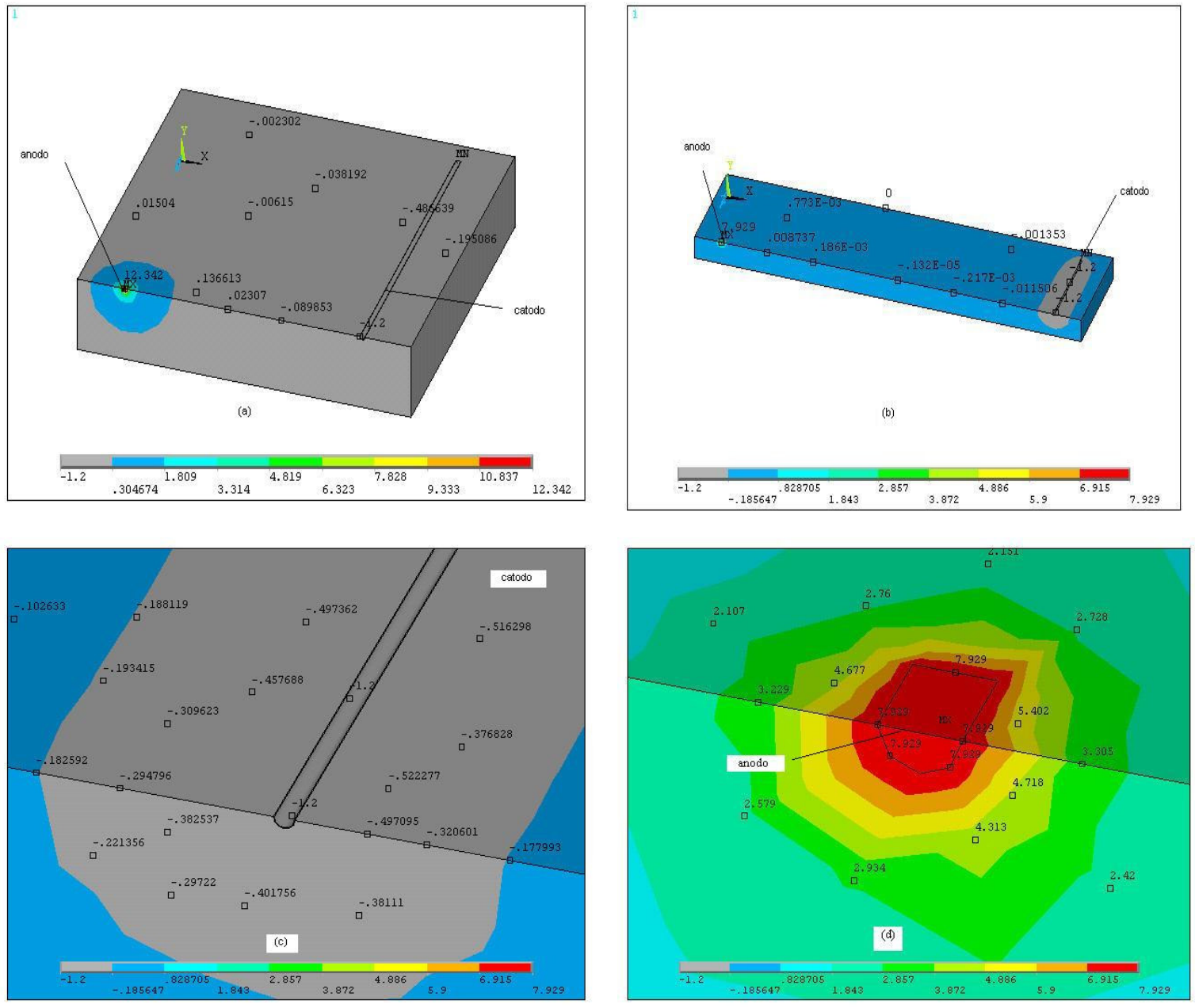

Figura 4. Diagrama da distribuição de potencial no sistema: (a) combinação dos parâmetros $(-1,-1,+1,+1,-1)$ da $7^{\text {a. }}$ linha; (b) combinação dos parâmetros $(-1,-1,-1,+1,+1)$ da $13^{\text {a. }}$ linha; (c) destaque na distribuição de potencial no catodo para $13^{\text {a. }}$ linha; (d) destaque na distribuição de potencial no anodo para a $13^{\mathrm{a}}$ linha.

Logo, apesar da resistividade influenciar a distribuição de potencial de acordo com alguns autores $[10,14,16]$, neste trabalho pode ser verificado que existe efeito significativo ao analisar-se a interação entre os parâmetros independentes. Segundo as observações realizadas para os resultados das simulações feitas, uma maior variação na distribuição de potencial no sistema pode ser atribuída à interação dos parâmetros, pois se observou que:

- Comparando os resultados das simulações da $1^{\mathrm{a}}$. linha (maior distribuição de 
potencial) e $8^{\mathrm{a}}$ (menor distribuição de potencial), neste caso, os resultados se apresentam em forma inversa. Pode-se considerar que a interação entre resistividade do eletrólito, diâmetro do duto, posição do anodo e potencial do catodo mostram grande influência na distribuição de potencial, sendo que estes parâmetros não são comuns a estas linhas. Por outro lado, todos estes parâmetros estão no limite inferior no planejamento fatorial que corresponde à $1^{\mathrm{a}}$. linha e no limite superior para a $8^{\mathrm{a}}$. linha.

- Da mesma forma, realizando a comparação dos resultados das simulações da $1^{\mathrm{a}}$. e $7^{\mathrm{a}}$. linhas, que mostraram distribuições de potencial semelhantes (maior distribuição), verifica-se que a interação de alguns parâmetros influencia na maior distribuição de potencial. Pois estas linhas possuem em comum parâmetros tais como: resistividade do solo, diâmetro do duto e distância entre catodo e anodo.

- Finalmente, através da observação dos resultados da distribuição de potencial que correspondem às simulações da $1^{\mathrm{a}}$ e $13^{\mathrm{a}}$ linhas, que apresentaram distribuição de potencial semelhante nas proximidades do anodo (maior distribuição), pode-se afirmar que esta semelhança pode estar relacionada com o efeito da interação entre resistividade do solo, diâmetro do duto e posição do anodo, sendo estes parâmetros comuns a estas linhas. 

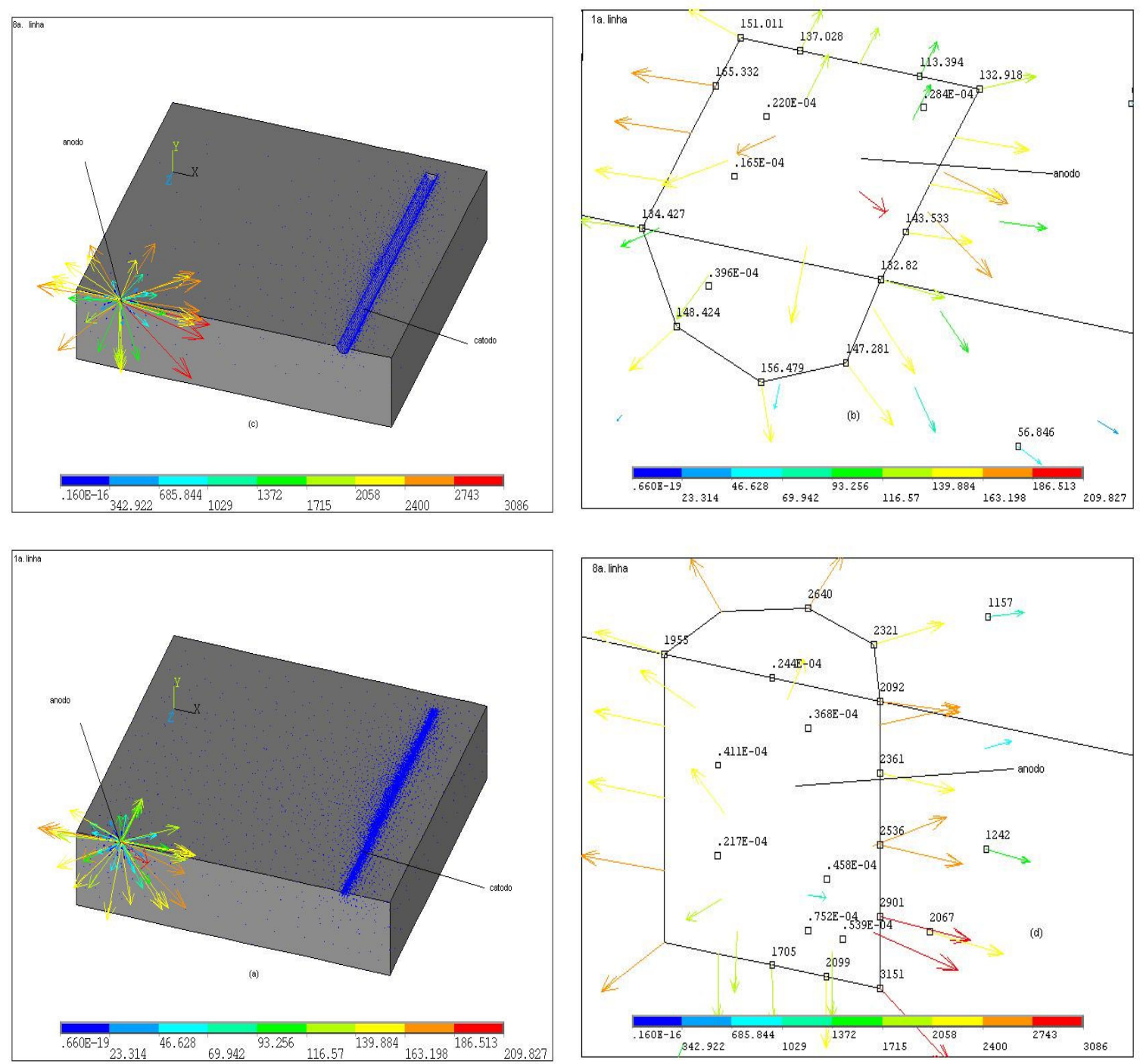

Figura 5. Diagrama do vetor gradiente do campo elétrico (V/m): (a) corresponde à 1 a linha; (b) corresponde ao anodo da $1^{\text {a }}$ linha da Tabela 9; (c) corresponde à $8^{\text {a }}$ linha; (d) corresponde ao anodo da $8^{\mathrm{a}}$ linha da Tabela 9.

Segundo as discussões realizadas anteriormente, observou-se que a resistividade do solo, diâmetro do duto, posição do anodo e potencial do catodo são parâmetros influenciadores na distribuição de potencial do sistema, como se observou nas simulações das linhas 1 e 8, as quais apresentaram respectivamente, maior e menor distribuição de potencial. Também se realizou a simulação do potencial em função da distância entre anodo e catodo, e considerou-se o potencial no ponto médio destas distâncias. Baseado neste resultado foi realizado o planejamento fatorial. Outro parâmetro independente importante é a distância 
entre catodo e ânodo: pode-se dizer que influencia consideravelmente na distribuição de potencial, como foi verificado nos resultados da simulação nos caminhos lineares, que correspondem às linhas 7 e 11 [15]. Os resultados que pertencem a estas linhas foram os mais destacáveis, confirmando assim as nossas afirmações.

Para complementar este trabalho, também foi realizada a simulação do vetor gradiente do campo elétrico $(\mathrm{V} / \mathrm{m})$ : para as linhas correspondentes 1 e 8 , estes resultados mostraram distribuições de potenciais diferentes, como já foi analisado anteriormente. Pois, o vetor de campo elétrico indica a direção em que é necessário deslocar-se para obter a mais rápida variação do potencial, sendo que, o resultado deste trabalho concorda com o resultado de Gasper [16].

Nas Fig. 5 (a) e (b), que correspondem à $1^{\mathrm{a}}$ linha, observa-se que no anodo a maior intensidade do gradiente do campo elétrico foi de 209,83 V/m (segundo a escala de cores), que está na direção do anodo para o catodo. Nas Fig. 5 (c) e (d), que correspondem à $8^{\mathrm{a}}$ linha, também no anodo, a maior intensidade do gradiente do campo elétrico foi de $3086 \mathrm{~V} / \mathrm{m}$ (segundo a escala de cores), na direção do anodo para o catodo. É de observar que o resultante do gradiente do campo elétrico para ambos os sistemas encontra-se na direção do anodo para o catodo. Também se verifica que os vetores do gradiente do campo elétrico do anodo são maiores que os do catodo, para ambos os sistemas. Justifica-se este fato, devido ao potencial do anodo ser maior que o potencial do catodo.

No presente trabalho, a prova de que a corrente flui do anodo para o catodo, se baseia no seguinte fato: "O sentido da corrente é no sentido do campo elétrico". Isto pode ser verificado, pois a resultante do campo elétrico está no sentido de anodo para o catodo e conseqüentemente a corrente dirige-se para o catodo, tal como é mostrado nas Fig. 5 (b) e (d), que apresentam o anodo ampliado e o sentido dos vetores, com os valores do vetor gradiente do campo elétrico em vários pontos. 


\section{Conclusão}

Neste trabalho realizado sobre a simulação de proteção catódica por corrente impressa, foi utilizada a equação de Laplace, pois no modelo construído não se utilizou uma fonte de corrente, como também, o eletrólito foi considerado homogéneo, e no modelo considerado a corrente de proteção não foi considerada como condição de contorno. Por outro lado, pode-se ressaltar ainda que a execução da simulação numérica em todas as áreas, se torna útil, devido ao fato de se poder realizar uma análise qualitativa e quantitativa dos parâmetros envolvidos. Através da análise do resultado neste trabalho, verificou-se que a interação dos quatro primeiros parâmetros independentes, ou seja, resistividade do eletrólito, diâmetro do duto, posição do anodo e potencial do catodo, foram os grandes influenciadores da maior distribuição de potencial do sistema modelado. Também foi verificado que o parâmetro independe da distância entre catodo e anodo, quando considerada menor, provoca uma maior distribuição de potencial do sistema. A preocupação em analisar a maior distribuição do potencial em torno do anodo foi devida ao fato de que, se esta distribuição fosse muito grande, poderia polarizar o catodo e, desta forma, poderia comprometer a proteção do sistema. Observou-se portanto, que o método de elementos finitos é um método eficiente para predizer o desempenho de sistemas de proteção catódica, avaliando o impacto de certas variáveis que dificilmente podem ser observadas em situações reais.

\section{Agradecimentos}

À Fundação Araucária pelo financiamento do projeto "Proteção catódica de gasodutos e simulação numérica por elementos finitos" e também pelo apoio financeiro para a participação no XVI SIBAE 2004 e ao CNPq pela bolsa de Iniciação Científica.

\section{Referências}

1. A.C. Dutra, Proteção catódica - Técnica de combate à corrosão, $3^{\mathrm{a}}$ ed., Editora Interciência, Rio de Janeiro, 1999.

2. V. Gentil, Corrosão, $4^{\mathrm{a}}$ ed., Livro Técnico Científico, Rio de Janeiro, 2003. 
3. M.G. Fontana, Corrosion Engineering, $3^{\mathrm{a}}$ ed., McGraw-Hill International Editions, New York, 1986.

4. P.A. Schweitzer, Corrosion and Corrosion Protection Handbook, M. Dekker, Inc., $2^{\text {nd }}$ ed., New York, 1989.

5. J.A.F. Santiago, J.C.F. Telles, Int. J. Num. Methods Eng. 40 (1997) 26112627.

6. J.A.F. Santiago, J.C.F. Telles, Adv. Eng. Software 30 (1999) 663-671.

7. W. Sun, Appl. Math. Modeling 20 (1996) 823-828.

8. N.G. Zamani, J.F. Forter, A.A. Mufti, Int. J. Num. Methods Eng. 23 (1986)1295-1311.

9. $\quad$ N.G. Zamani, J.M. Chuang, Optimal Control Appl. Methods 8 (1987) 339350.

10. R. Adey, J. Baynham, Computer Simulation as an Aid to CP System Design and Interference Prediction. Disponível em:

Www.beasy.com/images/pdf/publications/ papers/ceocor.pdf.

11. D.P. Riemer, M.E. Orazem, Models for cathodic protection of multiple pipeline with coating holidays, Proceedings of the CORROSION 99, NACE, p. 66-81, 1999.

12. R.A. Adey, Computer simulation as an aid to corrosion control and reduction, Proceedings of the CORROSION 99, NACE, p. 99-115, 1999.

13. S.L.D.C. Brasil, J.C.F. Telles, L.R.M. Miranda, Corrosion 56 (2000) 11801187.

14. S.L.D. Carvalho, J.C.F. Telles, L.M.R. Miranda, Computer Modeling in Corrosion, R.S. Munn (Ed.), ASTM STP 1154, Philadelphia, 1992, p. 277291.

15. F.O.R. Staroin, Simulação numérica da proteção catódica por corrente impressa de duto enterrado, Dissertação de mestrado, Prog. de PósGraduação em Eng. e Ciência de Materiais, Ponta Grossa-Brasil, 2003.

16. R.G. Kasper, M.G. April, Electrogalvanic Finite Element Analysis of Partially and Protected Marine Structures, NACE, v. 39, 1983. 